\title{
MATHEMATICAL MODELS FOR QUANTIFYING COLLECTIVE CELL BEHAVIOUR
}

\author{
STUART T. JOHNSTON
}

(Received 7 November 2017; first published online 20 February 2018)

2010 Mathematics subject classification: primary 60J70; secondary 35Q92, 62F15.

Keywords and phrases: approximate Bayesian computation, collective cell behaviour, mathematical model, cell migration, cell proliferation, random walk, wound healing, scratch assay.

Collective behaviour is critical to a variety of biological and ecological processes, including tumour invasion, wound healing and the spread of invasive species. Mathematical modelling techniques provide an opportunity for obtaining insight into the underlying mechanisms governing collective behaviour. Mathematical models can be interfaced with experimental data to enhance quantitative information obtained from experiments. The aim of this thesis is two-fold. First, to investigate the application of mathematical models to experimental data to obtain robust estimates of the parameters governing collective behaviour. Second, to develop novel continuum mathematical descriptions of individual-based models of birth, death and movement.

For the first part of this thesis, I begin by examining the application of a well-known mathematical model of cell motility and cell proliferation, the Fisher-Kolmogorov model, to IncuCyte $\mathrm{ZOOM}^{\mathrm{TM}}$ assay data for a prostate cancer (PC-3) cell population [5]. Standard techniques used to interpret IncuCyte $\mathrm{ZOOM}^{\mathrm{TM}}$ assay data do not provide insight about the individual contributions of cell motility and cell proliferation to the overall migration of the cell population. I find that by combining experimental measurements of the evolution of the position of the leading edge of the cell population and the evolution of the cell density away from the leading edge, I am able to obtain unique estimates of the cell diffusivity, cell proliferation rate and cell carrying capacity density. Furthermore, I am able to quantify how these parameters are influenced by the presence of varying concentrations of epidermal growth factor.

Next, I investigate whether the position of the leading edge of a cell population in a scratch assay can be combined with an appropriate mathematical model to provide unique estimates of the cell motility rate and the cell proliferation rate [7]. Leading

Thesis submitted to Queensland University of Technology in February 2017; degree approved on 4 August 2017; supervisors Matthew Simpson and Scott McCue.

(C) 2018 Australian Mathematical Publishing Association Inc. 
edge data is a commonly reported experimental measurement from scratch assays, which are typically interpreted in a qualitative fashion or with a quantitative technique that does not isolate the individual roles of motility and proliferation. I implement a lattice-based random walk model of motility and proliferation, mimic the geometry of a scratch assay and combine this mathematical model with experimental leading edge data through an automated edge detection algorithm. I find that, provided the difference in the time scales of cell proliferation and cell motility is accounted for, this technique produces unique estimates of the cell diffusivity and cell proliferation rate.

I then consider an approximate Bayesian computation (ABC) parameter recovery approach and examine which experimental measurements from a scratch assay provide the most information about the cell diffusivity and cell proliferation rate parameters [8]. ABC techniques provide parameter distributions rather than point estimates and hence contain quantitative information about the uncertainty associated with the parameter estimates. I find that an $\mathrm{ABC}$ approach provides parameter estimates that are consistent with previously obtained parameter estimates for 3T3 fibroblasts. Furthermore, I find that measuring the number of cells present within the assay and the pair correlation function provides sufficient information to obtain robust parameter estimates.

For the final piece of work associated with the first part of this thesis, I extend my $\mathrm{ABC}$ technique to examine the influence of experimental design choices on parameter estimates [4]. There is significant variation in the experimental design of scratch assays presented in the literature. Specifically, the number and timing of experimental observations captured, as well as whether the scratch assay contains one front or two opposingly directed fronts, varies throughout the literature. These design choices are not discussed and the influence of the choices has not been quantified. I find that capturing two experimental observations, after the initial observation, is sufficient to obtain robust parameter estimates. Additionally, I find that a scratch assay with a single front contains less uncertainty about the recovered parameters, compared to a scratch assay with two opposingly directed fronts.

These first four pieces of work highlight the importance of selecting an appropriate parameter recovery framework, which consists of a mathematical model, an experimental measurement or summary statistic and a parameter calibration technique. Throughout these pieces of work I demonstrate that making naïve framework choices does not necessarily elucidate the individual roles of cell motility and cell proliferation in the collective spreading of a cell population. Furthermore, mathematical modelling provides insight into how to refine the framework choices made such that the information obtained about cell motility and cell proliferation is maximised. In combination, these four pieces of work provide guidance on potential model and parameter recovery technique choices for given types of experimental data.

For the second part of this thesis, I first examine a corrected mean-field description of a lattice-based model of two interacting subpopulations, where the agents in each subpopulation undergo birth, death and movement events [6]. Standard mean-field descriptions for such models have been presented previously but provide inaccurate 
approximations of the average discrete behaviour outside limited parameter regimes. My investigation provides guidance about the choice of mean-field description required in a suite of parameter regimes for a number of case studies inspired by cell biology experiments.

Next, I present a novel interpretation of a lattice-based random walk model of birth, death and adhesive movement. Instead of considering the average occupancy of each lattice site, as is typical for both mean-field and corrected mean-field descriptions, I consider the lattice as a combination of groups of contiguous occupied sites and contiguous vacant sites [2]. I find that modelling the dynamics of these groups, referred to as chains and gaps, respectively, provides a more accurate description of the average discrete behaviour than either mean-field or corrected mean-field descriptions. Furthermore, this novel description contains a measure of the spatial clustering present in the discrete model.

I then extend the random walk model of birth, death and movement to incorporate competitive and/or co-operative mechanisms, where there is a negative or positive benefit associated with belonging to the bulk population. Such mechanisms arise throughout cell biology and ecology [1]. I derive the mean-field description of this random walk and analyse the long-time predictions in a suite of parameter regimes. I find that the inclusion of competitive and/or co-operative mechanisms results in a variety of interesting behaviour, depending on the relative rates of birth, death and movement. For example, I observe the Allee effect, where growth is negative below a threshold density, as well as travelling wave solutions that contain sharp fronts and shocks.

The final study in the second part of this thesis examines an extension of the chainand-gap description that can be used to model processes containing moving fronts, as well as competitive and/or co-operative mechanisms [3]. As such mechanisms give rise to more interesting behaviour than standard models of birth, death and movement, the question of whether the population persists or becomes extinct is critical. I find that the extended chain-and-gap description accurately predicts the long-time persistence or extinction of the population in all parameter regimes, as well as providing an accurate estimate of the rate of invasion of the population. Furthermore, the description provides details of the spatial clustering within moving fronts.

These second four pieces of work provide detailed information about the continuum approximations required to accurately reflect the average behaviour of discrete individual-based models of collective behaviour. The type of continuum approximation depends on the mechanisms present in the discrete model. In particular, if the combination of mechanisms and mechanism rates results in significant spatial clustering due to nearest-neighbour effects, typical approximations are inaccurate. As the chain-and-gap description accurately describes these cases, the combination of these four pieces of work provides a framework for informing the choice of continuum approximation for a particular discrete model.

In conclusion, I detail the contributions of each of the eight publications to the overall literature and provide discussion regarding potential avenues for future work 
related to collective behaviour. Overall, the work contained in this thesis provides a framework for modelling collective behaviour with random walks. In particular, I detail techniques for implementing an appropriate continuum description of random walks with a suite of mechanisms and parameter regimes, and methods for applying these random walk models of collective behaviour to relevant experimental data to obtain meaningful quantitative information.

\section{References}

[1] S. T. Johnston, R. E. Baker, D. L. Sean McElwain and M. J. Simpson, 'Co-operation, competition and crowding: a discrete framework linking Allee kinetics, nonlinear diffusion, shocks and sharpfronted travelling waves', Sci. Rep. 7 (2017), 42134.

[2] S. T. Johnston, R. E. Baker and M. J. Simpson, 'Filling the gaps: a robust description of adhesive birth-death-movement processes', Phys. Rev. E 93(4) (2016), 042413.

[3] S. T. Johnston, R. E. Baker and M. J. Simpson, 'A new and accurate continuum description of moving fronts', New J. Phys. 19(3) (2017), 033010.

[4] S. T. Johnston, J. V. Ross, B. J. Binder, D. L. Sean McElwain, P. Haridas and M. J. Simpson, 'Quantifying the effect of experimental design choices for in vitro scratch assays', J. Theoret. Biol. 400 (2016), 19-31.

[5] S. T. Johnston, E. T. Shah, L. K. Chopin, D. L Sean McElwain and M. J. Simpson, 'Estimating cell diffusivity and cell proliferation rate by interpreting IncuCyte $\mathrm{ZOOM}^{\mathrm{TM}}$ assay data using the Fisher-Kolmogorov model', BMC Syst. Biol. 9(38) (2015), 12 pages.

[6] S. T. Johnston, M. J. Simpson and R. E. Baker, 'Modelling the movement of interacting cell populations: a moment dynamics approach', J. Theoret. Biol. 370 (2015), 81-92.

[7] S. T. Johnston, M. J. Simpson and D. L. Sean McElwain, 'How much information can be obtained from tracking the position of the leading edge in a scratch assay?', J. R. Soc. Interface 11(97) (2014), 20140325.

[8] S. T. Johnston, M. J. Simpson, D. L. Sean McElwain, B. J. Binder and J. V. Ross, 'Interpreting scratch assays using pair density dynamics and approximate Bayesian computation', Open Biol. 4(9) (2014), 140097.

STUART T. JOHNSTON, School of Engineering,

University of Melbourne, Parkville, Victoria 3010, Australia

e-mail: stuart.johnston@unimelb.edu.au 